\title{
What exactly is central to the role of central neuroplasticity in persistent pain?
}

\author{
Terence J. Coderre ${ }^{a-c}$ and Joel Katz ${ }^{\mathrm{d}, \mathrm{e}}$ \\ aPain Mechanisms Laboratory, Clinical Research Institute of Montreal; \\ 'Centre de recherche en sciences neurologiques et departement de \\ médecine, Université de Montréal; and 'Department of Psychology, McGill \\ University, Montreal, Quebec H2W 1R7, Canada; dDepartment of \\ Psychology and Acute Pain Research Unit, The Toronto Hospital; and \\ -Departments of Behavioral Science and Anaesthesia, University of Toronto, \\ Toronto, Ontario M5S 2C4 Canada. \\ coderrt@ircm.umontreal.ca; www.ircm.umontreal.cal; \\ j.katz@utoronto.ca; www.utoronto.ca/
}

Abstract: The commentaries on our target article have raised important issues about central neuroplasticity and its role in persistent pain states. Some suggest that central neuroplasticity plays nothing more than a minor role in persistent pain, while others argue that persistent pain depends critically on peripheral inputs for its maintenance. Some stress that persistent pain relies to a large extent on changes in the brain and on centrifugal inputs from brain to spinal cord, whereas others argue that it depends on alterations in inhibitory as well as excitatory systems. We attempt to address each of the commentators' points, while defending our position that central neuroplasticity is critical to pathological persistent pain states.

Whether one calls it central hyperexcitability, sensitization, or neuroplasticity, the critical role of changes in central nervous system (CNS) function in persistent pain have been stressed both in our own target article and in the others in this issue of $B B S$. It is clear from the commentaries that the concept of noxious stimulus- or injuryinduced central changes has captured the attention of pain researchers world-wide. What is also clear is that there are many ideas and opinions about the nature of these changes and the extent to which they contribute to the pathophysiology of persistent pain. In an effort to integrate the large amount of material provided in the many insightful commentaries, we would like to draw the reader's attention to specific themes that have arisen, as well as to outline our own view on them. Despite the disparate views, we believe the commentaries have one thing in common. They all ask: What is the principal role of central neuroplasticity in persistent pain? Most agree that central changes play a role in pain processing, but some argue that peripheral pathology is central to its expression, and thus to persistent pain. Others argue that although central changes are key players in pathological pain, a greater emphasis should be placed on changes in the brain and the influence of the brain on spinal cord. Finally, still others point out, as we have, that central changes are important, but that the importance of changes in inhibitory rather than excitatory mechanisms must be stressed.

Determining the relative contribution of central changes and peripheral inputs is critical to answering the question of whether central neuroplasticity contributes to persistent pain. The issues raised in the commentaries can be condensed into four key questions. (1) Does central neuroplasticity exist? (2) If it exists, what role does it play in persistent pain in animal models or human clinical pain? (3) Are peripheral inputs more important than central changes for the expression of persistent pain? (4) Can neuroplasticity exist in the absence of continued or ongoing peripheral inputs? Most of the commentators seem to accept that central neuroplasticity exists. It would be difficult to ignore the growing body of experimental evidence demonstrating several forms of central neuroplasticity including wind-up, dorsal horn neuronal sensitization, and receptive field expansions, as well as hyperexcitable flexion reflexes and nerve injury-induced sprouting and the production of dark neurons in dorsal horn. However, it is possible to argue, as do Cleland \& Gebhart, that central neuroplasticity plays only an insignificant role in animal and human experimental hyperalgesia models. Marchettini et al. add that what occurs in animal experimental models may have little to do with what happens in human cases of chronic clinical pain. Furthermore, it is also possible to argue, as do both Devor and Gracely, that although central plasticity exists, from a treatment perspective it may be more appropriate to target the peripheral pathology that maintains it rather than the central site where it occurs. On the other hand, there is support from Jancsó et al. and from Willis to suggest that central sensitization can be maintained in the absence of continued sensory input. Hu \& Sessle go on to suggest that central sensitization in trigeminal nociceptive pathways may depend on an unmasking or strengthening of convergent inputs.

Cleland \& Gebhart have come to a different conclusion based on additional studies and their appreciation of certain critical technical limitations. However, these additional studies do not rule out a role for central neuroplasticity in hyperalgesia. Thus, the studies of Puig and Sorkin (1996), Dubner and Ruda (1992), and Woolf et al. (1992) indicate that peripheral injuries lead to changes in afferent activity, neuromediator release, and structural alterations that are present during both the development and the maintenance of hyperalgesia associated with these injuries. In each of these cases, however, the hyperalgesia-inducing injury is also associated with prolonged peripheral tissue changes. Thus, it is not unreasonable to expect central changes to accompany both phases of the response. It is important to realize that central neuroplasticity and continued inputs from the periphery are not mutually exclusive. However, when ongoing peripheral inputs are present, they obscure our ability to demonstrate that hyperalgesia is in part due to central changes. Even when using so-called direct methods (i.e., preinjury and postinjury block), results can be confounded if the injury is intense enough to produce prolonged peripheral changes. Thus, if intense peripheral inputs outlast a preinjury block then the block is unlikely to produce pre-emptive effects. On the other hand, if an important consequence of central sensitization is to amplify peripheral inputs, then eliminating peripheral inputs with a postinjury block may often eliminate nociceptive responses and hyperalgesia. These results do not rule out central neuroplasticity; they only stress that in many instances pain behaviour requires peripheral input to be fully expressed, a conclusion that we have espoused in our target article. On 
more technical grounds, we find it surprising that Cleland \& Gebhart accept that effective anesthesia was produced by $50 \mu \mathrm{l}$ of lidocaine in the study of Dallel et al. (1995) whereas they discount the anesthetic effect with a much larger volume $(150 \mu \mathrm{l})$ of the longer lasting anesthetic bupivicaine in the study of Coderre et al. (1990). We were also somewhat surprised that a 50\% reduction in withdrawal latency, significant at a p-value of $<0.01$, was deemed only weakly significant in the study of Coderre and Melzack (1985), while a stated nonsignificant trend in the study of McCall et al. (1996) was interpreted as significant by Cleland \& Gebhart.

Marchettini et al. are more receptive to the concept of central plasticity, but they remain doubtful that it plays a role in persistent human pain states. They correctly point out that there are examples of chronic clinical pain for which there are no experimental animal models. We agree that clinical and experimental pain are not identical, but we maintain that insights into the pathophysiology of clinical pain can be derived from experimental models. Even animal models as simple as the mouse tail-flick test have provided considerable information about the analgesic efficacy of various pharmaceutical compounds. It is true, however, that we are in need of additional animal models of chronic pain with demonstrated validity for human pain conditions. It should be noted that in human experimental trials, phenomena such as wind-up (Price et al. 1994) and hyperexcitability of flexion reflexes (Dahl et al. 1992) have been demonstrated and probably play a role in clinical pain. The fact that many human conditions - e.g., arthritis, soft tissue injury, and procedures such as nerve biopsies - do not lead to chronic pain in the absence of persistent injury is not proof that central plasticity does not exist, but only reinforces the point that there are fortunately other physiological mechanisms at work that undermine its expression and long-term survival. Unfortunately, there are probably other cases where these protective mechanisms are unable to overcome the negative consequences of central neuroplasticity or peripheral sources of pathology.

Marchettini et al. may be right that genetic differences may play a key role in determining who is at risk for developing chronic pain, as has been well articulated by Devor and Raber (1990), and that human cognition can lead to suppression or augmentation of pain perception and can produce variability in human pain behaviour that is less likely or dramatic in animal models. We agree with Backonja, who argues that neuropathic pain is an intricate condition that cannot be explained by neuroplasticity alone. A complex interaction between peripheral pathology, CNS changes, and reactive emotive processing must be taken into account.

Both Devor and Gracely make the point that central neuroplasticity is selective and not striking in intensity and hence that targeting the peripheral pathology would be a more effective treatment. We agree that there has recently been an overemphasis on central mechanisms in the explanation of persistent pain states. However, until recent years there was barely a recognition that central plasticity played any role at all in persistent pain. Over the last several years there has been a growing recognition that central plasticity exists and plays a role in persistent pain. In the introduction of any new idea, there is a tendency to overstate its significance. Throughout the target article we made an effort, as did Gracely et al. (1992), to bring peripheral mechanisms back into the picture and to focus on the interaction between peripheral and central mechanisms of persistent pain. We stress that in some clinical pain states peripheral mechanisms may dominate, whereas central mechanisms may be more important in others. It is true that there has been an explosion in the literature on novel centrally acting agents, such as $\mathrm{N}$-methyl-D-aspartate (NMDA) antagonists for the treatment of persistent pain. Perhaps for many patients such a treatment is neither appropriate nor effective. However, there may also be a group of patients who do not respond when peripheral pathologies are targeted but are responsive to such a central treatment (Backonja et al. 1994; Eide et al. 1994; Kristensen et al. 1992; Nikolajsen et al. 1996). Rather than seeing central treatments as targeting an all-inclusive, final common pathway, it may be more instructive to think of them as alternatives, to be used when agents targeting the peripheral pathology are ineffective. It is for this reason that we must not be too quick to dismiss new treatment possibilities.

Generally, we agree with Devor and Gracely that in many instances central sensitization is probably not so much pathology as a natural response of the CNS to peripheral pathology. It is clear, however, that CNS neurons are capable, in some instances, of developing pathological characteristics (i.e., epileptic foci). Because neurons involved in pain transmission share neurochemical similarities with those involved in epilepsy, including glutamate activity at NMDA receptors, it is not inconceivable that, as suggested in the commentary by Backonja, such central pathologies may contribute to some persistent pain states. Furthermore, as discussed by Jancsó et al., peripheral nerve injuries result in progressive structural changes in pain transmission pathways, including transganglionic degeneration of C-fiber primary afferents, sprouting of large fibers into substantia gelatinosa, and a reorganization of spinal dorsal horn neuronal connections, all central changes which could contribute significantly to the development of persistent pain after nerve injury. Also, as described by $\mathbf{H u}$ \& Sessle, central sensitization that is evident in trigeminal nociceptive pathways may depend on an unmasking or strengthening of convergent inputs. Since neuroplasticity is more effectively induced by noxious deep inputs, Hu \& Sessle also suggest this may explain why greater sensory disturbances occur after injury to deep tissues than after injury to cutaneous tissues.

The question remains as to whether central sensitization can be sustained in the absence of continued inputs from the periphery. Gracely has given evidence of persistent clinical pain states where inputs from the periphery are necessary, and in our target article we have given clinical examples in which it is possible that inputs from the periphery are not necessary to sustain central hyperexcitability. Although Gracely does not rule out the possibility, Cleland \& Gebhart and Devor are more skeptical, and Ursin, in contrast, supports even the concept of selfsustaining positive feedback loops. Jancsó et al. argue that sustained central sensitization is possible, because after intracisternal injection of capsaicin a mechanical hyperalgesia develops in the skin area that becomes completely insensitive to further noxious chemical stimulation. Furthermore, both Jancsó et al. and Willis point out that capsaicin injection to the skin produces a significant hyperalgesia that is dependent on central sensitization. Hole et al. and Birbaumer \& Flor further support this concept 
and argue that sustained central sensitization could be explained by mechanisms similar to those involved in learning and memory processes.

Hole et al. review the data from their own as well as four independent studies that suggest that phenomena such as long-term potentiation may exist in spinal cord dorsal horn. In relation to the mechanisms triggering sensitization, Willis describes how the sensitization of spinothalamic tract neurons induced by intradermal capsaicin is mimicked by excitatory mediators such as glutamate and substance $\mathrm{P}$ and blocked by both NMDA and NKI receptor antagonists, as well as inhibitors of various protein kinases. Jancsó et al. also refer to evidence that perineural treatment with capsaicin produces transganglionic degeneration of C-fiber primary afferents and extensive sprouting of large fibers within the substantia gelatinosa, resulting in alterations in connectivity similar to those produced by peripheral nerve sections. This evidence is all quite significant, since, as we point out in our target article, the hyperalgesia produced by capsaicin typically long outlives the duration of its initial afferent barrage.

Devor raises an important question: What is there about the theory of central sensitization that would predict stamping in of an ingrown toenail, but not an episiotomy scar? Our model proposes that reactivation of a somatosensory pain memory requires a drive and that this drive may originate in the periphery, DRG, spinal cord, or brain. In the case of amputation, we need to consider whether the loss of normal sensory nerve impulses (deafferentation) that follows amputation in some way plays a role in either the stamping in process, the re-activation stage, or both. We have previously argued that the interruption of afferent input associated with amputation or deafferentation may facilitate the central neural changes that contribute to the formation of pain memories by removing normal inhibitory control mechanisms (Coderre et al. 1993). There are, however, examples of pain memories recurring in the absence of obvious deafferentation, although it is true that the example of postepisiotomy pain raised by Devor is not among them. Thus, cardiac pain had been referred to the site of a compression fracture in the upper back (Henry \& Montuschi 1978) and pain in response to stimulation of the nasal mucosa may be referred to teeth that had recently been filled (Hutchins \& Reynolds 1947; Reynolds \& Hutchins 1948). It appears then that deafferentation may not necessarily play a role in the stamping in of a pain.

What about the role of deafferentation in the reactivation of an established pain trace? The most important difference between a phantom ingrown toenail and an episiotomy scar is that the former has no peripheral referent, since the body part has been surgically removed. We need to consider some of the less obvious implications of this fact for the reactivation of a pain trace after amputation. In addition to the loss of afferent input that results from amputation of a body part, there is also a loss of visual, tactile, and proprioceptive information related to the limb; it can no longer be seen, touched, or felt. What is the effect of this loss of information on the perception of a phantom? We would argue that the cortical and subcortical influences that normally inhibit established pain traces may be further reduced by the absence of information from sense modalities that might otherwise confirm or disconfirm the percept (e.g., of a painful ingrown toenail) arising from the periphery. Following amputation, the likelihood of re- activating a pain memory that had a visual component (e.g., ingrown toenail) is increased because the potential inhibitory effect of vision has also been removed. In general, as the number of modalities involved in the pre-amputation pain experience increases (and thus the more sources of potential feedback are removed), so does the probability of re-activating a past pain once the limb has been removed. This could occur because there are fewer senses available to provide a reality-based check (i.e., exert an inhibitory influence) on the perceptual processes generating the phantom (Katz 1993).

Implicit in any discussion of memory is the assumption that the CNS has been changed as a function of prior experience. Although we did not explicitly refer to learning in our target article, we agree with Hole et al., Birbaumer \& Flor, and Watkins \& Maier that learning processes must underlie expressions of new behaviour. Perhaps the most fascinating example of this in the phantom limb pain literature is learned paralysis (Ramachandran 1994), in which the brain has learned that an immobile phantom hand cannot be moved. An extremely creative solution is to use mirrors to trick the brain into thinking that the phantom is moving by having the amputee look into a mirror while his contralateral intact hand is positioned to coincide spatially with the felt position of the phantom hand. When the amputee attempts to carry out the same movement with both hands while looking at the phantom (i.e., reflection of the intact hand), the sight of the hand moving determines the ultimate perception, and the amputee feels as if the once paralyzed hand is now moving freely. This experiment highlights the dominant role of vision over other sensory modalities in circumstances involving exteroceptive sensibility. There are other examples of this intermodal integration and perceptual dominace of vision (Katz 1993). $\mathrm{Pa}$ tients undergoing brachial plexus or spinal local anaesthestic blocks and patients with complete brachial plexus avulsions or spinal cord transections all report vivid phantom limbs that are felt to be coincident with the postion of the real limb as determined by sight. However, when a patient's deafferented limb is moved from one position to another with eyes closed, the felt position of the phantom corresponds to the last seen position of the real limb. When patients open their eyes, the phantom is reported to fuse with the new position of the real limb as perceived by sight. These examples demonstrate that we are dealing with a perception system that is even more complex than the traditional supraspinal pain signalling system envisioned by Hardcastle in her commentary.

There is no doubt, as Benedetti argues, that psychological factors play a role in the perception of pain after surgery. It is for this reason that we routinely administer the multidimensional McGill Pain Questionnaire in our studies of pre-emptive analgesia (Katz et al. 1994; Katz et al. 1996a; Katz et al. 1992). We have also administered other measures of psychological and emotional functioning, including anxiety and depression (Kavanagh et al. 1994). Although we and others have found that pre-operative administration of analgesics or local anesthetic agents can pre-empt postoperative pain, we have yet to find any differential effects of these psychological factors on analgesic requirements after surgery. A recent prospective follow-up study of patients who had undergone lateral thoracotomy showed that $52 \%$ of patients reported daily or weekly pain of moderate intensity approximately 1.5 years after surgery (Katz et al. 
1996b). The interesting finding was that postoperative pain within 6 hours of surgery was the only significant predictor of long-term pain. In contrast, pre- and postoperative measures of anxiety and depressive symptomology were not predictive, suggesting that these psychological factors did not differentially influence the experience or reporting of pain. While psychological factors are important, too much emphasis on them may blind clinicians and researchers to other potentially significant predictors of longterm pain in this case, intense postoperative pain.

As noted above, the model we developed in the target article allows for the maintaining drive to originate from brain regions either directly or indirectly involved in the processing of nociceptive information. As we did not emphasize this possibility in the target article, we welcome the commentary by Watkins \& Maier who bring into focus a novel line of pain research involving brain-to-spinal cord circuitry. Whereas it is well established that noxious peripheral stimulation leads to a sensitization of spinal cord neurons, Watkins \& Maier introduce the novel concept that centrifugal brain to spinal cord pathways allow descending inputs from brain centers such as the nucleus raphe magnus to facilitate nociceptive processing in spinal cord as well.

Along similar lines, Benedetti discusses the role of nociceptive emotional integration in the limbic system and its relation to pathological pain; and Ursin raises the possibility of the brain generating and maintaining sensitization in the absence of peripheral input. Elsewhere, we have outlined a mechanism through which cognitive and affective processes associated with higher cortical and limbic centers may alter phantom limb sensations via either brain-to-brain or brain-to-spinal cord circuitry (Katz 1996). As an example of the latter, phantom limb pain intensity may be modulated by higher brain centers involved in cognitive and affective processes via a multisynaptic network of descending inputs that impinges on preganglionic sympathetic neurons in the lateral horn of the spinal cord. These descending inputs would subsequently produce diffuse peripheral autonomic discharge and activation of primary afferent fibers located in stump neuromas. This activity would, in turn, project to spinal cord dorsal horn neurons subserving the amputated limb and to rostral brain structures where the impulses contribute to the perception of pain. Consistent with the learning model outlined by Birbaumer \& Flor in their commentary, we have pro- posed (Katz 1993) that through repeated activation, neural circuitry is strengthened among brain regions subserving cognitive, affective, and sensory processes. Hence phantom limb sensations and pain may be triggered by thoughts and feelings in the absence of primary afferent feedback from peripheral structures.

Clarke is right in pointing out that our target article concentrated heavily on excitatory mechanisms rather than inhibitory mechanisms. It is quite true that failure of inhibitory mechanisms could underlie some forms of pathological pain. Indeed, this possibility is discussed in greater detail in the accompanying target articles of DickENSON and Wiesenfel-Haldin et al. Furthermore, many of the most effective treatments that clinicians use today (opiates, $\alpha 2$-adrenergic agonist and tricyclic antidepressants) are based on the enhancement of inhibitory systems rather than suppression of exictatory systems. These treatments are available today because of the research efforts into inhibitory systems (endogenous opioids, descending control circuits, etc.) in the 1970s and early 1980s. This is precisely why it is necessary to develop a better understanding of the excitatory mechanisms that play a role in persistent pain. The accelerated pace of research into excitatory mechanisms (neuropeptides, excitatory amino acids) in the late 1980s and 1990s promises to deliver novel clinical treatments that will give us additional options to alleviate persistent pain.

To respond to a specific comment from Clarke about referred pain: we did not mean to suggest that referred pain relies on tonic inputs from the area of referral but rather that referred pain is influenced by additional inputs from the area of referral. Unless one proposes an axon-reflex-like peripheral response in the referred area, this conclusion is necessary to explain how referred pain sensations can be reduced by local anesthesia of the referred area. In response to Marchettini et al., we wish to point out that we did not intend to equate phantom limb pain with referred pain and that in the final analysis we described phantom limb pain as pain that is projected (not referred) to the amputated region. Last, in response to Devor's stated paradox about $A \beta$-fiber input producing both increased inputs and counterstimulation-induced decreased inputs: (1) There is no requirement that central sensitization reliably render $A \beta$-fiber input painful, and (2) counterstimulation is most often produced by heterosegmental sensory input.

\section{No brain, no pain}

\section{Zsuzsanna Wiesenfeld-Hallin \\ Karolinska Institute, Department of Medical Laboratory Sciences and Technology, Section of Clinical Neurophysiology, S-14186 Huddinge, Sweden.zswh@bimd01.hs.sll.se}

Abstract: The theme of my target article was dysfunction of inhibition in the spinal cord as an important factor in the development of chronic pain states. Some commentaries focused on the role of more central mechanisms and the limited usefulness of animal models for understanding mechanisms of human pain. More specific comments concerned the roles of GABA and cholecystokinin in pain control.

The commentaries can divided into broad categories dealing with the following issues:

1. Spinal mechanisms are inadequate to describe the pathophysiology underlying chronic pain because not enough attention has been focused on conditioning and learning (Birbaumer \& Flor, Hole et al., Watkins \& Maier), central effects of infection/inflammation (Watkins \& Maier), cortical functions, and emotions (Hardcastle). Pain facilitating circuits descending from the brain to the spinal cord may have a key role in the organization of 
response to pain. Furthermore, it is unquestionable that mechanisms rostral to the spinal cord are of great importance in pain perception and suffering. However, the emphasis of this review was on spinal mechanisms because a better understanding of these should offer great hope for therapies, with minimal side effects. Of course, pains that involve structures central to the spinal cord, such as thalamic or idiopathic pains, cannot be treated in the same way as pains arising from peripheral or spinal pathology.

2. Animal studies are of limited use in understanding clinical human conditions (Marchettini et al.). It is undoubtedly true that human pain and suffering reported verbally cannot be duplicated, for obvious reasons, in animals. However, after careful analysis of animal behavior one can certainly detect abnormalities that might be relevant to the human experience. More specifically, abnormal painlike behaviors that can be alleviated by drugs (WiesenfeldHallin et al. 1997) and other therapies, such as spinal cord stimulation (Stiller et al. 1996), that are useful in humans for pain relief should be considered as evidence for the relevance of the animal model. In discussing the reduced analgesic effect of opiates reported by some clinicians, but not others, Marchettini et al. write that making "comprehensive hypotheses on neuropathic pain without considering divergent reports seems simplistic." However, such reports are considered under section 4.1 (para. 2) in the target article, and even in our own animal studies we have found reduced potency of intrathecal morphine following axotomy, rather than a total lack of effect (Xu \& WiesenfeldHallin 1991). If the potency of opiates is indeed reduced in neuropathic pain, physicians need to consider carefully whether "analgesia" after large doses has a large sedative component. The sedative effect of systemic morphine in experimental pain states can be analyzed with careful behavioral techniques (Xu et al. 1992). Furthermore, comprehensive hypothesis about neuropathic pain is proposed, but there is a suggestion that there may be some common mechanisms. As pointed out by Siddall, different models of neuropathy (axotomy vs. partial nerve injury) may have very different mechanisms. It has recently been demonstrated that there are considerable differences among various models of partial nerve injury (Kim et al. 1997).

3. A number of neurotransmitter systems are involved in the mediation of chronic pain. The target article concentrates on two systems: $\gamma$-amino butyric acid (GABA) and cholecystokinin (CCK). The role of GABA in the mediating of chronic pain states may involve both the GABA-A and GABA-B receptor in both spinal and trigeminal pathways (Hu \& Sessle). Furthermore, dysfunction of the GABAergic system may differ in pain states with varying etiologies (Omote, Siddall).

CCK's role as an antianalgesic or antiopioid is now widely accepted, on the basis of pioneering studies from a number of laboratories (Han, Noble et al., Watkins \& Maier). Although the functional role for such a mechanism from the evolutionary point of view may seem counterintuitive (Clarke), there are very interesting hyptheses about a role for CCK as a negative feedback control for endogenous opioids (Han). In spite of a great deal of research on the antiopioid action of CCK, however, the precise mechanism of action is not well understood. There is general agreement about an interaction between the CCK and the mu-opioid receptor, but there is divergence of opinion about whether there is also an important interaction between the $\kappa$ (Han) or $\delta$ opioid (Benoliel et al. 1991) receptors. An important therapeutical possibility for CCK antagonists would also be to increase the analgesic effect of inhibitors of enkephalindegrading enzymes, which increase the potency of endogenous opioids (Noble et al.). Like all neuroactive substances, CCK has a number of functions in the central and peripheral nervous systems, including being a potent anxiogenic when applied exogenously (Benedetti). In fact, highly selective CCK-B antagonists have been found to have a potent anxiolytic action in rodents (Hughes et al. 1990). It is tempting to suggest that clinically useful CCK antagonists could have a double beneficial effect, by reducing the sensory and affective/anxiogenic aspects of pain.

\title{
Sympathetic contribution to pain - need for clarification
}

\author{
Helmut Blumberg, a Ulrike Hoffman, ${ }^{b}$ Mohsen Mohadjer, a \\ and Rudolf Scheremeta \\ a Department of Neurosurgery, University of Freiburg, 79106 Freiburg, \\ Germany; ${ }^{b}$ Department of Anesthesiology, University of Freiburg, 79106 \\ Freiburg, Germany, boeger@nz11.ukl.uni-freiburg.de
}

Abstract: Certain patients with a possible contribution of the sympathetic system to pain may not fit the definition of complex regional pain syndromes (CRPS), which raises the question of terminology for those patients. To further clarify the relationship between the sympathetic system and pain, apart from the need for placebo studies, there remains an urgent need for a satisfactory definition of the criteria for a complete sympathetic block. It also remains uncertain whether a change in the discharge pattern of sympathetic fibres underlies the changes in sympathetic organ function, often found in patients with CRPS.

\section{R1. Introduction}

Our target article received a number of thoughtful and well structured commentaries. It was not possible, nor was it our intention, to clarify all aspects of the relationship between the sympathetic system and pain. The commentaries accordingly show that there is a need to clarify the contribution of the sympathetic system to pain states.

\section{R2. Definition}

We were aware of recent changes in nomenclature from the international association for the study of pain regarding the "complex regional pain syndrome" (CRPS) (Merskey \& Bogduk 1994). Nevertheless, for one group of patients we found it reasonable at this stage of the discussion to continue using the term "reflex sympathetic dystrophy" 